\title{
El capital social en la Encuesta de Calidad de Vida en el Trabajo*
}

\section{Félix Requena Santos}

Universidad de Santiago de Compostela. Departamento de Sociología

Campus Sur. 15782 Santiago de Compostela

frequena@usc.es

\section{Resumen}

Este artículo señala la multidimensionalidad del concepto de capital social. A pesar de que existen importantes diferencias en el consenso de una definición de capital social, es fundamental buscar indicadores empíricos para su medida. En este trabajo se muestran y se analizan las dimensiones del capital social sobre las que pregunta la Encuesta de Calidad de Vida en el Trabajo que realiza el Ministerio de Trabajo y Asuntos Sociales, y se comparan las dimensiones de la definición de capital social que propone la OCDE, comparando cuáles de los indicadores que propone la OCDE se encuentran en la ECVT. También se pone de manifiesto la utilidad social del concepto de capital social como predictor de la calidad de vida.

Palabras clave: capital social, redes sociales, calidad de vida, mercado de trabajo.

\section{Abstract. Social Capital in the Spanish Quality of Working Life Survey}

This article point out multidimensionality of social capital concept. Although there are great differences for a consensual social capital definition, it is important to look for empirical indicators for its measurement. This work analyses social capital questions in the Spanish Quality of Working Life Survey. Theses questions are compared with OECD proposed, and its level of comparability. As well as this paper shows the utility of social capital concept as a quality life predictor.

Key words: social capital, social networks, quality of life, labour market.

\section{Sumario}

1. Introducción 4. El capital social como factor explicativo

2. La Encuesta de Calidad de Vida de la calidad de vida en el trabajo en el Trabajo (ECVT)

5. Conclusiones

3. El capital social en la ECVT

Bibliografía

* Una versión previa de este artículo fue presentada en la Conferencia Internacional de la OCDE y la Oficina de Estadística de Gran Bretaña sobre la Medida del Capital Social, en Londres, 25-27 de septiembre de 2002. 


\section{Introducción}

En los últimos años se viene usando con gran profusión el concepto de capital social, sin embargo, su cuantificación y medida se presentan como una cuestión todavía difícil. Esta dificultad se agrava con el problema de su definición ${ }^{1}$. Respecto a la definición del concepto, existen dos tendencias fundamentales, que corresponden con dos usos diferentes del término «capital social».

El primero, representado entre otros por Putnam (1993; 2000) y Fukuyama (1995; 2000), concibe el capital social como una cualidad de los grupos (o incluso de la sociedad en su conjunto). Es una concepción en parte cultural y en parte social. Incluye aspectos tales como el sometimiento al imperio de la ley, la integración social y la confianza tanto en los otros como en las instituciones. Hace referencia a lo positivo que tiene el bien colectivo que suponen los valores compartidos con los demás. Esta concepción es la que hace referencia al capital social como el conjunto de normas o valores informales que comparten los miembros de un grupo y que permiten su cooperación. Cuando los miembros del grupo tienen el convencimiento de que los demás se van a comportar con formalidad y honestidad, nace la confianza entre ellos (Fukuyama, 2000: 29).

De esta forma, las normas asociadas al capital social son la veracidad para con los miembros del grupo, el cumplimiento de las obligaciones, la reciprocidad y el cumplimiento de los compromisos. Esto hace que cada miembro del grupo sepa qué lugar ocupa en el seno del grupo, de forma que disminuye la competencia individual entre sus miembros porque cada uno sabe lo que opinan los otros, y, al mismo tiempo, aumenta la competencia del conjunto del grupo porque permite coordinar las acciones del grupo frente al exterior.

Además, de esta forma, el ambiente relajado dentro del grupo, los contactos fáciles entre sus miembros y los constantes intercambios de información generan un mayor grado de innovación y adaptación del grupo. Esto es muy importante en los grupos que se dedican al tratamiento de la información y el conocimiento, en los que se exige de ellos que para ser competitivos sean imaginativos e innovadores. La imaginación y la innovación quedan considerablemente reducidas si los miembros del grupo están en constante competencia entre ellos o están más pendientes de cuál es su posición respecto a los otros que en su trabajo innovador y creativo.

Bien es verdad que es perfectamente posible formar grupos que funcionen sin capital social, es decir, grupos que estén cohesionados mediante mecanismos formales tales como contratos, jerarquías y constituciones. Pero las normas informales reducen mucho lo que los economistas llaman "costes de transacción», es decir, los costes de controlar, contratar, obligar y vigilar el cumpli-

1. A pesar de los excelentes trabajos que recientemente se han realizado con la intención de clarificar una definición de capital social. Sin embargo, dichos esfuerzos sólo ponen de manifiesto que se trata de un concepto al que todavía se le dan diversas concepciones. Véase Herreros Vázquez (2002). 
miento de todas las normas formales. Al mismo tiempo que reduce los niveles de espontaneidad e innovación de los miembros del grupo.

El segundo uso, representado entre otros por Burt (2000; 2001), concibe el capital social como el valor de las relaciones sociales de los individuos. Esta concepción ha sido la principal en toda la bibliografía del apoyo social. Consiste en que las relaciones de unos con otros son una fuente material de información, de recursos y de ayuda material. Esta concepción es la más formal de las dos, también llamada "teoría de los recursos sociales». De forma similar, en el análisis de las organizaciones, las relaciones se han visto como una fuente de poder porque pueden proporcionar beneficios desde el punto de vista del control de la información. Este punto de vista tiene sus raíces en una larga tradición sociológica que ve la posición del actor en una red social como determinante para sus oportunidades y limitaciones. Es decir, enfatiza el carácter estructural que tiene el capital social. Hace referencia al bien individual que supone "mis conexiones pueden ayudarme».

Esta segunda acepción ve el capital social como el conjunto de oportunidades relacionales que tiene un actor social respecto a la situación de la acción que está desarrollando en la sociedad. Desde este punto de vista, el capital social son las ventajas competitivas que un actor tiene para poner en práctica el capital humano que posee, en función de la posición social que ocupa dentro de un amplio conjunto de redes sociales.

Estos dos usos del concepto de capital social reflejan dos niveles de análisis: el del individuo y el del grupo. Pero los grupos que estudiamos no son universos, sino que son grupos concretos que están insertos en sus propios entornos sociales. A continuación vamos a examinar la posibilidad de medición del capital social en los entornos laborales, en los grupos de trabajo; para ello analizaremos la capacidad que tiene la Encuesta de Calidad de Vida en el Trabajo (ECVT) para proporcionar información sobre el capital social que tienen los ocupados españoles en su entorno laboral.

Este artículo analiza las posibilidades para medir el concepto de capital social que tiene la ECVT y compara las definiciones anteriores, que se pueden obtener en mayor o menor medida con la encuesta, con la definición de capital social que propone la Organización para la Cooperación y el Desarrollo Económico (OCDE).

\section{La Encuesta de Calidad de Vida en el Trabajo (ECVT)}

En 1999, el Ministerio de Trabajo y Asuntos Sociales puso en marcha el proyecto de Encuesta de Calidad de Vida en el Trabajo (ECVT) ${ }^{2}$. Su objetivo es tener una herramienta para obtener, anualmente, información sustantiva sobre

2. La Encuesta de Calidad de Vida en el Trabajo está englobada en el Plan Estadístico Nacional que elabora el Instituto Nacional de Estadística. Los datos de esta encuesta están a disposición pública a través de la Subdirección General de Estadísticas Sociales y Laborales del Ministerio de Trabajo y Asuntos Sociales. 
las relaciones sociales, la situación, las actitudes y los valores hacia el trabajo de los ocupados españoles. El núcleo de la encuesta es doble: por un lado, obtener información sobre las circunstancias reales de las actividades y relaciones que tienen lugar en el trabajo y, por otro, obtener información sobre la percepción personal que los trabajadores tienen del entorno y las actividades que realizan de forma cotidiana.

La ECVT tiene una periodicidad anual y está basada en un muestreo trietápico estratificado por comunidades autónomas y tamaño de municipio, cuyo ámbito es toda España. El universo es la población ocupada de dieciséis y más años de ambos sexos que reside en viviendas familiares. El tamaño muestral es de $N=6.020$ ocupados. Las entrevistas se realizan en los hogares de los trabajadores para que éstos no estén sometidos a posibles presiones derivadas del entorno de las empresas. El error de muestreo para el total nacional, en el caso más desfavorable y para un intervalo de confianza del $95,5 \%(2 \sigma)$, es de $\pm 1,4 \%{ }^{3}$. Los datos de la estructura ocupacional que muestra la ECVT son concordantes con la Encuesta de Población Activa.

Uno de los objetivos principales de la encuesta es obtener información sobre la satisfacción de los distintos componentes que forman el trabajo cotidiano. La satisfacción en el trabajo es muy importante para la actividad productiva. Estar o no satisfecho con las tareas que se realizan está muy relacionado con la cantidad y la calidad de la productividad del trabajador. En la encuesta, la satisfacción se toma como un concepto multidimensional: hace referencia a un amplio conjunto de situaciones y aspectos que rodean al puesto de trabajo, que van desde las relaciones con los compañeros, los superiores, etc. hasta la percepción de las condiciones de trabajo, el estrés, el cansancio, etc ${ }^{4}$. Se trata, así, de obtener información sobre las relaciones laborales y la calidad de vida en el trabajo, y relacionarlas con la estructura familiar y el entorno social de los trabajadores.

La ECVT se estructura en varios bloques:

1) El estudio de la calidad de vida en el trabajo.

2) La situación real en el puesto de trabajo, estudiando elementos tales como la división, la organización, etc. del trabajo.

3) La estructura familiar del ocupado.

4) Los valores, las actitudes y la percepción del trabajo.

5) Datos sociodemográficos del ocupado.

Dentro del conjunto de preguntas relativas a las relaciones en el puesto de trabajo y de los valores y actitudes, la ECVT realiza preguntas que proporcionan varias de las dimensiones del capital social. Además, sensible a la cuestión del funcionamiento del capital social en el entorno laboral, en 2001 el equipo de diseño añadió en el cuestionario un grupo de preguntas relativas a la con-

3. Los detalles sobre el diseño muestral de la encuesta pueden verse en MTAS, 2001, (http://www.mtas.es/Estadisticas/ECVT/Welcome.htm)

4. Para más detalles sobre la estructura y las posibilidades de la ECVT, véase Requena, 2000. 
fianza, las relaciones, la compromiso, la comunicación, la influencia; elementos que proporcionan una visión del capital social en el entorno del trabajo.

\section{El capital social en la ECVT}

\section{El concepto de capital social usado en la ECVT}

El capital social es un concepto multidimensional. Las perspectivas con las que se ha definido (Herreros Vázquez, 2002) van desde la implicación en las relaciones sociales y las conexiones derivadas de la pertenencia a redes sociales (Burt, 2000; 2001), hasta la participación en asociaciones que generan compromiso civil (Putnam, 1993; Putnam, Leonardi y Nanetti, 1993), o la generación de confianza generalizada en los otros (Coleman, 1988; Brehm y Rahn, 1997)

La importancia del concepto de capital social se debe a que aglutina varios conceptos sociológicos importantes, tales como apoyo social, integración o cohesión social. A su vez, estos conceptos tienen repercusiones importantes, tanto en la vida política (Brehm y Rahn, 1997), como en la económica (Knack y Keefer, 1997; Beugelsdijk, Groot y Van Schaik, 2002). Las organizaciones también funcionan mejor si tienen una buena dosis de capital social (Lin y otros, 2001).

El capital social y los procesos de confianza que genera son esenciales para el buen funcionamiento de las relaciones de mercado sobre las que se basan las instituciones económicas (Fukuyama, 1995). Del mismo modo, las transacciones económicas como los contratos o la búsqueda de empleo son mucho más eficientes cuando están insertas en redes sociales (Granovetter, 1985; Requena, 1991; De Graff y Flap, 1988). Un buen ejemplo es cómo las redes sociales son un buen potenciador de los movimientos en el mercado de la innovación tecnológica, como puede verse desde el Silicon Valley en California (Saxenian, 1994) o en los distritos industriales del norte de Italia (Pyke y otros, 1990). Según estos análisis, el capital social es un buen lubricante para las industrias de alta tecnología o la moda. Desde este punto de vista, el enfoque del capital social ayuda a formular nuevas estrategias para el desarrollo.

La ECVT utiliza el concepto de capital social en su sentido más amplio, es decir, el que incluye aspectos tales como el sometimiento a las normas, la integración social y la confianza en los otros y en las instituciones sociales, así como las relaciones sociales. Se trata, por tanto, de un conjunto de normas y valores informales que comparten los miembros de un grupo y que permiten que haya cooperación. Estas normas y valores compartidos generan confianza entre ellos. Es decir, es el conjunto de relaciones cooperativas entre actores sociales que facilitan la solución a los problemas de acción colectiva.

Esta definición es muy similar a la que utiliza la OCDE: Networks together with shared norms, values and understandings that facilitate co-operation within or among groups (redes junto con las normas y los valores compartidos y comprendidos que facilitan la cooperación en y entre los grupos) (OCDE, 2001), 
dado que las relaciones (redes) cooperativas implican unas normas y unos valores compartidos.

\section{Preguntas de capital social en la ECVT}

El concepto de capital social tiene múltiples aplicaciones operativas para el análisis de las organizaciones sociales y, concretamente, las empresariales. La empresa es una organización que necesita de la cooperación de sus miembros para lograr el éxito de sus actividades. Por este motivo, a la ECVT le interesa estudiar cómo determinados aspectos del capital social (confianza, compromiso, relaciones con los otros) inciden en el desarrollo de las actividades que se realizan en el interior de las empresas y organizaciones, de forma que producen mayor o menor bienestar en el trabajo.

Aunque el objetivo principal de la ECVT es el estudio de la calidad de vida en el trabajo, de sus datos también se puede estudiar un amplio conjunto de cuestiones relativas a la población trabajadora. De este modo, es posible estudiar algunos elementos de la definición de capital social de la OCDE, tales como las relaciones sociales, la confianza en los otros, las normas compartidas, la participación en la comunidad.

La ECVT cubre casi todas las dimensiones del capital social que propone la OCDE (véase tabla 1). Sin embargo, conviene recordar que están dirigidas sólo a la población ocupada y no a la población en general. Además, la ECVT ha considerado algunas dimensiones extra del capital social, tales como la comunicación y la influencia, por supuesto, siempre en el contexto de las organizaciones empresariales. Consideramos que estos dos elementos tienen importancia como canalizadores (comunicación) y como resultado o consecuencia del capital social.

\section{Medida de las dimensiones de capital social en la ECVT}

Como ocurre en la mayoría de las encuestas, las preguntas que se diseñan para realizarlas se hacen ad hoc y de una forma pragmática de acuerdo con las necesidades y los objetivos de la encuesta. A la ECVT le ocurre esto. Sus preguntas han sido diseñadas para estudiar los procesos de satisfacción y la calidad de vida en el trabajo. Sin embargo, en su diseño se ha tenido en cuenta la importancia que tienen los componentes del capital social en la satisfacción y la calidad de vida en el trabajo. Aunque no es expresamente una encuesta para estudiar el capital social, se puede estudiar de una forma relativamente satisfactoria el capital social de la población ocupada. Esto hace que la mayoría de las referencias a las relaciones sociales, la confianza, la participación y el compromiso estén referidos siembre a los superiores y subordinados, a los compañeros de trabajo y al entorno de la empresa u organización.

Dada la multidimensionalidad del concepto de capital social, es necesario una amplia variedad de indicadores para medirlo. Así, un buen índice de capital social será el que cubra una gran cantidad de aspectos relativos a todas sus dimensiones. La ECVT cubre la mayoría de esas dimensiones. 
Tabla 1. Matriz del capital social. Comparación de las definiciones de la ECVT y la OCDE.

\begin{tabular}{|c|c|c|c|c|c|}
\hline Tema & Aspecto & Medida & V en el cuestionario & Def. ECVT & Def. OCDE \\
\hline \multirow[t]{4}{*}{ Confianza } & \multirow[t]{3}{*}{ Confianza en los otros en general } & Confianza en los superiores & V305 & $\bullet 1$ & - \\
\hline & & Confianza en los compañeros de trabajo & V306 & $\bullet 1$ & - \\
\hline & & Confianza en los subordinados & V307 & $\bullet 1$ & • \\
\hline & $\begin{array}{l}\text { Confianza en grupos concretos } \\
\text { y en instituciones }\end{array}$ & Confianza en el lugar de trabajo & V316 & $\bullet 1$ & - \\
\hline \multirow[t]{5}{*}{ Compromiso } & \multirow[t]{5}{*}{ Compromiso con la empresa } & Trabajar más para ayudar a mi empresa & V196 & • & • \\
\hline & & $\begin{array}{l}\text { Los problemas de mi empresa son como } \\
\text { si fueran míos }\end{array}$ & V201 & $\bullet$ & $\bullet$ \\
\hline & & Estoy orgulloso de trabajar para mi empresa & V197 & $\bullet$ & • \\
\hline & & $\begin{array}{l}\text { En mi empresa existe colaboración porque hay } \\
\text { una jerarquía que hace que se cumplan } \\
\text { las tareas (inversa) }\end{array}$ & V313 & $\bullet 1$ & $\bullet$ \\
\hline & & $\begin{array}{l}\text { En el trabajo, lo importante es cumplir } \\
\text { con las obligaciones del contrato, } \\
\text { extralimitarse significa que al final no te lo } \\
\text { agradecen y no sirve de nada (inversa) }\end{array}$ & V314 & $\bullet 1$ & • \\
\hline \multirow[t]{7}{*}{$\begin{array}{l}\text { Redes } \\
\text { informales }\end{array}$} & $\begin{array}{l}\text { Provisión de ayuda no pagada a amigos, } \\
\text { parientes, vecinos fuera del hogar }\end{array}$ & & & & $\bullet$ \\
\hline & \multirow[t]{3}{*}{$\begin{array}{l}\text { Recepción de ayuda de otras personas } \\
\text { de fuera del hogar }\end{array}$} & $\begin{array}{l}\text { Consiguió su trabajo actual a través de amigos, } \\
\text { conocidos o parientes }\end{array}$ & V30 & $\bullet$ & - \\
\hline & & $\begin{array}{l}\text { Consiguió su primer trabajo a través de amigos, } \\
\text { conocidos o parientes }\end{array}$ & V52 & $\bullet$ & $\bullet$ \\
\hline & & $\begin{array}{l}\text { Provisión de ayuda económica por parte } \\
\text { de los amigos cuando estaba en paro y no } \\
\text { cobraba desempleo }\end{array}$ & V63 & $\bullet$ & $\bullet$ \\
\hline & \multirow{3}{*}{$\begin{array}{l}\text { Otra ayuda o implicación social } \\
\text { con amigos, parientes, conocidos, } \\
\text { compañeros de trabajo }\end{array}$} & Relaciones de amistad en el trabajo & V308 & $\bullet 1$ & • \\
\hline & & Buenas relaciones con mis jefes & V142 & $\bullet$ & $\bullet$ \\
\hline & & Buenas relaciones con mis compañeros & V143 & $\bullet$ & • \\
\hline
\end{tabular}


Tabla 1. Matriz del capital social. Comparación de las definiciones de la ECVT y la OCDE (continuación).

\begin{tabular}{|c|c|c|c|c|c|}
\hline Tema & Aspecto & Medida & V en el cuestionario & Def. ECVT & Def. OCDE \\
\hline \multirow[t]{3}{*}{ Participación } & $\begin{array}{l}\text { Participación en grupos organizados } \\
\text { (religiosos, políticos, culturales, etc.) }\end{array}$ & $\begin{array}{l}\text { Miembro activo / no activo de organizaciones } \\
\text { voluntarias: } \\
\text { - Profesionales } \\
\text { - Ecologistas, medio ambiente } \\
\text { - Deportivas, recreativas } \\
\text { - Culturales, artísticas } \\
\text { - Religiosas } \\
\text { - Partidos políticos } \\
\text { - Benéficas, de ayuda } \\
\text { - Organizaciones de vecinos } \\
\text { - Sindicatos }\end{array}$ & $\begin{array}{l}\text { V206 } \\
\text { V207 } \\
\text { V208 } \\
\text { V209 } \\
\text { V210 } \\
\text { V211 } \\
\text { V212 } \\
\text { V213 } \\
\text { V216 a V219 }\end{array}$ & $\bullet$ & $\cdot$ \\
\hline & $\begin{array}{l}\text { Trabajo voluntario llevado a cabo } \\
\text { en esos grupos (tipos de grupos) }\end{array}$ & (Véase casilla superior) & & & $\bullet$ \\
\hline & $\begin{array}{l}\text { Vínculos dentro de los grupos } \\
\text { y entre los grupos }\end{array}$ & & & & • \\
\hline \multirow[t]{6}{*}{ Otros factores } & Bienestar & Tamaño de la vivienda & V176 & - & $\bullet$ \\
\hline & & Número de habitaciones de la vivienda & V177 & • & - \\
\hline & Bienestar subjetivo & Satisfacción con el trabajo & V92 & $\bullet$ & • \\
\hline & & Satisfacción con la vida que se tiene & V204 & • & • \\
\hline & & Nivel general de felicidad & V205 & • & • \\
\hline & Participación política & & & & $\bullet$ \\
\hline \multirow{2}{*}{\multicolumn{2}{|c|}{ Comunicación Comunicación en la empresa }} & Puede dar sus opiniones en la empresa & V131 & $\bullet$ & \\
\hline & & Conoce los objetivos de su empresa & V134 & • & \\
\hline \multirow[t]{3}{*}{ Influencia } & Participación en la empresa & Participa en las decisiones respecto a sus tareas & V116 & $\bullet$ & \\
\hline & & Mi jefe valora mis sugerencias & V132 & $\bullet$ & \\
\hline & & Puedo poner en práctica mis ideas & V111 & $\bullet$ & \\
\hline
\end{tabular}

Nota: 1. Disponible sólo en el cuestionario de 2001 y siguientes. El resto de las preguntas se realizaron en todas las oleadas. 
Veamos algunas de las cuestiones que miden estas dimensiones:

Confianza. Tiene dos aspectos, la confianza en los otros y la confianza en las instituciones (en nuestro caso en la empresa). Por un lado, la confianza en los otros se mide con la pregunta:

Muchas veces las personas que trabajan juntas en una empresa confían las unas en las otras porque funciona un conjunto de normas implícitas comunes. En cambio, otras veces, las personas no confían las unas en las otras y sólo colaboran cuando existen unas normas y unos reglamentos que se negocian y muchas veces se aplican a la fuerza.

¿Por favor, podría decirme si Vd., concretamente, confía mucho, bastante, poco, casi nada o nada en las siguientes personas de su empresa u organización?

- Confianza en mis superiores

- Confianza en mis compañeros

- Confianza en mis subordinados.

Éste es el indicador ${ }^{5}$ central de confianza de la ECVT. Cuando se necesita tener información sobre los diferentes tipos de "otros", esta pregunta proporciona información sobre los superiores, los compañeros y los subordinados. Sin embargo, el indicador se puede usar en conjunto - no con el interés de estudiar un tipo particular de posición en la empresa—, es decir, tomándolo como una pregunta que hace referencia general a la confianza en los otros. De hecho, los tres tipos aparecen correlacionados en la mayoría de los casos, con lo que al final, en la práctica, se puede resumir en un solo indicador: «confianza en los otros en el interior de la empresa u organización».

Correlaciones de las confianzas en los otros*

\begin{tabular}{llll}
\hline & $\begin{array}{l}\text { Confianza en } \\
\text { mis superiores }\end{array}$ & $\begin{array}{l}\text { Confianza en } \\
\text { mis compañeros }\end{array}$ & $\begin{array}{l}\text { Confianza en } \\
\text { mis subordinados }\end{array}$ \\
\hline Confianza en mis superiores & - & 0,506 & 0,531 \\
Confianza en mis compañeros & & - & 0,629 \\
Confianza en mis subordinados & & & - \\
\hline
\end{tabular}

* Calculada sólo con trabajadores asalariados: $N=4683$.

Todas las correlaciones son significativas al nivel 0,01 (bilateral).

Por otro lado, la confianza en las instituciones se mide en la ECVT con una pregunta relativa a la confianza en la empresa en la que se trabaja.

5. Esta pregunta sobre la confianza en los otros en la empresa se ha introducido en la ECVT a partir de 2001. 
Concretamente, la pregunta se formula como la confianza en los otros que pertenecen a la institución empresarial. La pregunta es la siguiente:

En general, ¿está Vd. muy de acuerdo, de acuerdo, indiferente, en desacuerdo o muy en desacuerdo con la siguiente afirmación?

- En mi empresa/organización las personas que trabajamos juntas confiamos las unas en las otras, porque así se hace el trabajo más fácil y mejor.

Sin embargo, esta pregunta es más un matiz de la confianza en los otros en el contexto del trabajo, que una pregunta sobre la confianza en la empresa como institución empresarial. Probablemente, la confianza en la empresa como institución se vea mejor en el conjunto de preguntas que la ECVT ha definido genéricamente como "compromiso» (véase tabla 1).

El compromiso, es decir, el hecho de sentirse obligado a cuidar de algo y verlo como algo propio, en este caso de la empresa. Este compromiso con la institución (la empresa) se ha medido con el siguiente grupo de preguntas:

En general, ¿está Vd. muy de acuerdo, de acuerdo, indiferente, en desacuerdo o muy en desacuerdo con la siguiente afirmación?

— «Estoy dispuesto a trabajar más de lo que debo para ayudar a triunfar a la empresa u organización para la que trabajo».

- «Los problemas de mi empresa son como si fueran míos».

— «Estoy orgulloso de trabajar para mi empresa».

Como han señalado Brehn y Rahn (1997), es interesante apreciar la correlación entre la confianza en los otros y la confianza en las instituciones. Así, las correlaciones entre la confianza en los otros (superiores y subordinados) y el compromiso con la empresa puede verse en el siguiente cuadro:

Correlaciones de las confianzas en los otros y el compromiso en la empresa*

\begin{tabular}{|c|c|c|c|c|c|c|}
\hline & (a) & (b) & (c) & (d) & (e) & (f) \\
\hline Confianza en mis superiores (a) & - & 0,506 & 0,531 & 0,311 & 0,413 & 0,296 \\
\hline Confianza en mis compañeros (b) & & - & 0,629 & 0,188 & 0,259 & 0,169 \\
\hline Confianza en mis subordinados (c) & & & - & 0,177 & 0,268 & 0,231 \\
\hline $\begin{array}{l}\text { Identificación con la empresa } \\
\text { (trabajar más) (d) }\end{array}$ & & & & - & 0,456 & 0,465 \\
\hline $\begin{array}{l}\text { Identificación con la empresa } \\
\text { (orgullo de empresa) (e) }\end{array}$ & & & & & - & 0,387 \\
\hline $\begin{array}{l}\text { Identificación con la empresa } \\
\text { (problemas de empresa) (f) }\end{array}$ & & & & & & - \\
\hline
\end{tabular}

* Calculada sólo con trabajadores asalariados: $N=4683$.

Todas las correlaciones son significativas al nivel 0,01 (bilateral). 
En la ECVT, aunque las correlaciones entre la confianza en los otros y el compromiso con la empresa son significativas estadísticamente, la correlación no es tan alta como señalan Brehm y Rahn (1997: 1014) cuando estudian la relación entre la confianza en las instituciones gubernamentales y la confianza en los otros $(0,63)$. Sin embargo, la correlación aumenta cuando se trata de la confianza en los superiores y el orgullo por trabajar en una empresa concreta $(0,41)$.

Las redes informales pueden obtenerse a través de las siguientes preguntas relativas a la recepción de ayuda por parte de otras personas de fuera del hogar, tales como amigos, conocidos, parientes o vecinos. Las preguntas hacen referencia a cómo consiguió su empleo (el empleo actual o el primer empleo) a través de amigos, conocidos, etc.; o bien, si obtenía ayuda financiera de amigos, etc. cuando estaba desempleado. También se pregunta por la implicación en relaciones de amistad con los compañeros de trabajo. Sin embargo, la ECVT no pregunta sobre la provisión de ayuda no pagada a los amigos, conocidos, etc.

La participación en la comunidad también es objeto de interés en la ECVT. Esta encuesta considera la participación en asociaciones un indicador de integración en la comunidad, lo que supone una cuestión importante para la calidad de vida de las personas en general y en particular en el puesto de trabajo. Se pregunta por la pertenencia como miembro y como miembro activo en una serie de organizaciones voluntarias. Sin embargo, aunque no se pregunta expresamente si se realiza trabajo no pagado en estas organizaciones, esta información puede quedar cubierta con el matiz que diferencia entre miembro activo y miembro no activo.

Obviamente, en la ECVT no se pregunta por la participación política. Sin embargo, sí existen varias preguntas relativas a la comunicación en la empresa y a la posibilidad de participación en las decisiones. Estos dos elementos, comunicación y participación, también habría que considerarlos como elementos del capital social. Así los ha entendido la ECVT.

\section{El capital social como factor explicativo de la calidad de vida en el trabajo}

A continuación, mostraremos un ejemplo de la utilidad del concepto de capital social en el estudio de la calidad de vida en el trabajo (Requena, 2003). Hay una asociación fuerte y significativa entre el capital social ${ }^{6}$ y el nivel de calidad

6. En este análisis (Requena, 2003), el capital social se midió con las siguientes variables: - Confianza: tengo [mucha, bastante, poca, casi nada, ninguna] confianza en mis superiores. En mi empresa u organización, las personas que trabajamos juntas confiamos unas en otras, porque así se hace el trabajo más fácil y mejor.

- Relaciones sociales: ¿por favor, podría decirme si Vd. mantiene fuertes relaciones de amistad con [todos, la mayoría, algunos, casi ninguno o ninguno] de sus compañeros de trabajo? (en el sentido de pedirles un favor importante, o salir a cenar o tomar algo con ellos y/o 
de vida $^{7}$ en el trabajo $(0,60)$. Con un análisis de regresión, se puede ver cómo el capital social es un buen factor explicativo de calidad de vida en el trabajo. La confianza, las relaciones sociales en el puesto de trabajo, el compromiso con la empresa u organización, la comunicación y las posibilidades de influencia en la realización del trabajo cotidiano son elementos que explican una gran proporción de la varianza total de calidad de vida que se puede tener en el puesto de trabajo. Así, los niveles de bienestar personal en el puesto de trabajo se incrementan cuando el trabajador goza de niveles mayores de confianza, relaciones, comunicación, compromiso e influencia en su trabajo.

En la tabla 2 se puede ver el modelo de regresión en el que, de forma consecutiva, se han ido añadiendo predictores sobre la calidad de vida en el trabajo. El modelo en su conjunto explica el 35,8 \% de la varianza de la calidad de vida en el trabajo. En cada interacción se añadía un grupo de variables en el modelo. Los grupos de variables fueron las siguientes: atributos personales, atributos familiares, atributos de la empresa u organización en la que trabaja el sujeto y atributos del puesto de trabajo y, por último, las dimensiones del capital social. Cuando se estudiaban de forma separada los primeros grupos de variables de atributos, todos ellos eran significativos para explicar la calidad de vida en el trabajo, sin embargo, cuando se añadía el capital social como predictor en los modelos de regresión, entonces la varianza explicada del modelo aumentaba el doble (2,01 veces). Por tanto, aun siendo muy importantes los atributos de los trabajadores, de los puestos de trabajo y de la empresa en la que se trabaja en la explicación de la calidad de vida laboral, sin embargo, el capital social ejerce una fuerza explicativa mucho mayor sobre la calidad de vida laboral.

En el modelo sobre la calidad de vida en el trabajo la fuerza de los predictores de atributos son diferentes. De esta forma, la edad, los años de educa-

sus parejas). En general, ¿cómo describiría Vd. las relaciones entre compañeros en su lugar de trabajo? [muy buenas, bastante buenas, ni buenas ni malas, bastante malas, muy malas]. - Compromiso: Estoy dispuesto a trabajar más de lo que debo para ayudar a triunfar a la empresa u organización para la que trabajo. Estoy orgulloso de trabajar para mi empresa u organización. Los problemas que tiene mi empresa u organización los considero como si fueran míos.

- Comunicación: Puedo dar mis opiniones sobre lo que respecta a mi trabajo.

- Influencia: En mi trabajo puedo poner en práctica mis ideas.

7. El índice de calidad de vida en el trabajo utilizado en la ECVT es un índice complejo que hace referencia exclusivamente al puesto de trabajo. Este indicador está construido de forma que incluye elementos subjetivos y elementos objetivos que afectan a la calidad de vida en el trabajo. Está construido otorgando un punto por cada situación que se produce en los empleados (situaciones objetivas): si puede trabajar con independencia; si no trabaja los fines de semana; si come en casa y no en su lugar de trabajo; si no tiene hacinamiento en su hogar. Situaciones subjetivas: si muestra un alto grado de satisfacción con el trabajo que realiza; si considera estimulante su ambiente de trabajo; si su trabajo no es agotador. Es una escala que varía de 0 a 7 según se trate de muy baja calidad de vida o de muy alta calidad de vida. El indicador se ha normalizado para que tome el valor de 0 a 10 , de forma que sea más fácil su comprensión. 
Tabla 2. Efectos sobre la calidad de vida en el trabajo.

\begin{tabular}{|c|c|c|c|c|c|}
\hline Variables independientes & Ec. $(1)$ & Ec. $(2)$ & Ec. $(3)$ & Ec. (4) & Ec. $(5)$ \\
\hline Correlación múltiple & 0,238 & 0,312 & 0,411 & 0,421 & 0,598 \\
\hline Constante & $\begin{array}{l}-0,596 \\
(-0,72) \\
\end{array}$ & $\begin{array}{l}-0,480 \\
(-0,53) \\
\end{array}$ & $\begin{array}{l}-2,192 \\
(-2,26)^{*}\end{array}$ & $\begin{array}{l}-2,985 \\
(-3,03)^{*}\end{array}$ & $\begin{array}{l}-1,209 \\
(-1,27) \\
\end{array}$ \\
\hline \multicolumn{6}{|l|}{ Variables personales } \\
\hline Edad & $\begin{array}{l}0,018 \\
(6,26)^{* * *}\end{array}$ & $\begin{array}{l}0,012 \\
(4,01)^{* * *}\end{array}$ & $\begin{array}{l}0,002 \\
(0,54)\end{array}$ & $\begin{array}{l}0,008 \\
(2,22)\end{array}$ & $\begin{array}{l}0,01 \\
(2,62)^{* *}\end{array}$ \\
\hline Varón & $\begin{array}{l}-0,264 \\
(-3,87)^{* * *}\end{array}$ & $\begin{array}{l}-0,173 \\
(-2,38)^{*}\end{array}$ & $\begin{array}{l}0,01 \\
(0,13)\end{array}$ & $\begin{array}{l}0,07 \\
(0,91)\end{array}$ & $\begin{array}{l}-0,46 \\
(-0,60)\end{array}$ \\
\hline Años de educación & $\begin{array}{l}0,076 \\
(8,83)^{* * *}\end{array}$ & $\begin{array}{l}0,052 \\
(5,49)^{* * *}\end{array}$ & $\begin{array}{l}0,015 \\
(1,57)\end{array}$ & $\begin{array}{l}0,011 \\
(1,18)\end{array}$ & $\begin{array}{l}0,011 \\
(1,21)\end{array}$ \\
\hline Ingresos & $\begin{array}{l}0,365 \\
(4,75)^{* * *}\end{array}$ & $\begin{array}{l}0,389 \\
(4,67)^{* * *}\end{array}$ & $\begin{array}{l}0,785 \\
(8,55)^{* * *}\end{array}$ & $\begin{array}{l}0,853 \\
(9,21)^{* * *}\end{array}$ & $\begin{array}{l}0,325 \\
(3,65)^{* * *}\end{array}$ \\
\hline Tamaño del hábitat & $\begin{array}{l}-0,045 \\
(-3,16)^{* *}\end{array}$ & $\begin{array}{l}-0,046 \\
(-3,03)^{* *}\end{array}$ & $\begin{array}{l}-0,05 \\
(-3,42)^{* *}\end{array}$ & $\begin{array}{l}-0,05 \\
(-3,41)^{* *}\end{array}$ & $\begin{array}{l}-0,46 \\
(-3,26)^{* *}\end{array}$ \\
\hline \multicolumn{6}{|l|}{ Variables de la empresa } \\
\hline Tamaño de la empresa & & $\begin{array}{l}-0,064 \\
(-4,42)^{* * *}\end{array}$ & $\begin{array}{l}-0,067 \\
(-4,69)^{* * *}\end{array}$ & $\begin{array}{l}-0,067 \\
(-4,73)^{* * *}\end{array}$ & $\begin{array}{l}-0,018 \\
(-1,34)\end{array}$ \\
\hline Industria & & $\begin{array}{l}1,164 \\
(6,45)^{* * *}\end{array}$ & $\begin{array}{l}0,965 \\
(5,43)^{* * *}\end{array}$ & $\begin{array}{l}0,927 \\
(5,24)^{* * *}\end{array}$ & $\begin{array}{l}0,829 \\
(4,95)^{* * *}\end{array}$ \\
\hline Construcción & & $\begin{array}{l}0,515 \\
(2,75)^{* *}\end{array}$ & $\begin{array}{l}0,580 \\
(3,14)^{* *}\end{array}$ & $\begin{array}{l}0,559 \\
(3,03)^{* *}\end{array}$ & $\begin{array}{l}0,568 \\
(3,27)^{* *}\end{array}$ \\
\hline Servicios & & $\begin{array}{l}0,956 \\
(5,48)^{* * *}\end{array}$ & $\begin{array}{l}0,719 \\
(4,19)^{* * *}\end{array}$ & $\begin{array}{l}0,675 \\
(3,95)^{* * *}\end{array}$ & $\begin{array}{l}0,422 \\
(2,60)^{* *}\end{array}$ \\
\hline Sector Privado & & $\begin{array}{l}-0,501 \\
(-5,44)^{* * *}\end{array}$ & $\begin{array}{l}-0,421 \\
(-4,58)^{* * *}\end{array}$ & $\begin{array}{l}-0,422 \\
(-4,61)^{* * *}\end{array}$ & $\begin{array}{l}-0,458 \\
(-5,46)^{* * *}\end{array}$ \\
\hline Realiza turnos & & $\begin{array}{l}-0,778 \\
(-9,43)^{* * *}\end{array}$ & $\begin{array}{l}-0,626 \\
(-7,69)^{* * *}\end{array}$ & $\begin{array}{l}-0,621 \\
(-7,66)^{* * *}\end{array}$ & $\begin{array}{l}-0,433 \\
(-5,75)^{* * *}\end{array}$ \\
\hline \multicolumn{6}{|l|}{ Variables entorno del trabajo } \\
\hline Horas de trabajo semanales & & & $\begin{array}{l}-0,05 \\
(-13,73)^{* * *}\end{array}$ & $\begin{array}{l}-0,047 \\
(-14,04)^{* * *}\end{array}$ & $\begin{array}{l}-0,042 \\
(-12,44)^{* * *}\end{array}$ \\
\hline Nivel de jerarquía & & & $\begin{array}{l}0,06 \\
(0,69)\end{array}$ & $\begin{array}{l}0,067 \\
(0,778)\end{array}$ & $\begin{array}{l}0,005 \\
(0,06)\end{array}$ \\
\hline Realiza supervisión & & & $\begin{array}{l}0,140 \\
(1,46)\end{array}$ & $\begin{array}{l}0,158 \\
(1,66)^{*}\end{array}$ & $\begin{array}{l}-0,111 \\
(-1,26)\end{array}$ \\
\hline Años de antigüedad en la empresa & & & $\begin{array}{l}0,006 \\
(1,49)\end{array}$ & $\begin{array}{l}0,007 \\
(1,72)^{*}\end{array}$ & $\begin{array}{l}0,004 \\
(0,93)\end{array}$ \\
\hline Condiciones de trabajo peligrosas & & & $\begin{array}{l}-0,257 \\
(-9,44)^{* * *}\end{array}$ & $\begin{array}{l}-0,249 \\
(-9,17)^{* * *}\end{array}$ & $\begin{array}{l}-0,187 \\
(-7,38)^{* * *}\end{array}$ \\
\hline
\end{tabular}


Tabla 2. Efectos sobre la calidad de vida en el trabajo (continuación).

\begin{tabular}{|c|c|c|c|c|c|}
\hline Variables independientes & Ec. $(1)$ & Ec. $(2)$ & Ec.(3) & Ec.(4) & Ec.(5) \\
\hline \multicolumn{6}{|l|}{ Variables entorno familiar } \\
\hline Cabeza de familia & & & & $\begin{array}{l}-0,175 \\
(-2,10)^{*}\end{array}$ & $\begin{array}{l}-0,007 \\
(-0,09)\end{array}$ \\
\hline Casado & & & & $\begin{array}{l}0,095 \\
(0,96)\end{array}$ & $\begin{array}{l}0,021 \\
(0,22)\end{array}$ \\
\hline Separado o divorciado & & & & $\begin{array}{l}0,198 \\
(1,18)\end{array}$ & $\begin{array}{l}0,135 \\
(0,86)\end{array}$ \\
\hline Viudo & & & & $\begin{array}{l}0,439 \\
(1,46)\end{array}$ & $\begin{array}{l}0,239 \\
(0,80)\end{array}$ \\
\hline Hijos a cargo & & & & $\begin{array}{l}-0,412 \\
(-4,66)^{* * *}\end{array}$ & $\begin{array}{l}-0,375 \\
(-4,50)^{* * *}\end{array}$ \\
\hline \multicolumn{6}{|l|}{ Variables de capital social } \\
\hline Confianza & & & & & $\begin{array}{l}0,261 \\
(5,86)^{* * *}\end{array}$ \\
\hline Relaciones & & & & & $\begin{array}{l}0,191 \\
(4,79)^{* * *}\end{array}$ \\
\hline Compromiso & & & & & $\begin{array}{l}0,317 \\
(8,85)^{* * *}\end{array}$ \\
\hline Comunicación & & & & & $\begin{array}{l}0,018 \\
(0,63)\end{array}$ \\
\hline Influencia & & & & & $\begin{array}{l}0,419 \\
(16,66)^{* * *}\end{array}$ \\
\hline $\begin{array}{l}\text { R-cuadrado } \\
\text { F del modelo }\end{array}$ & $\begin{array}{l}0,056 \\
50,437^{* * *}\end{array}$ & $\begin{array}{l}0,097 \\
37,843^{* * *}\end{array}$ & $\begin{array}{l}0,169 \\
47,616^{* * *}\end{array}$ & $\begin{array}{l}0,178 \\
38,432^{* * *}\end{array}$ & $\begin{array}{l}0,358 \\
69,707^{* * *}\end{array}$ \\
\hline $\begin{array}{l}\text { Comparación con el modelo a } \\
\text { Incremento en el R-cuadrado } \\
\text { Veces de incremento }\end{array}$ & & $\begin{array}{l}0,041 \\
1,73\end{array}$ & $\begin{array}{l}0,072 \\
1,74\end{array}$ & $\begin{array}{l}0,009 \\
1,05\end{array}$ & $\begin{array}{l}0,18 \\
2,01\end{array}$ \\
\hline
\end{tabular}

Fuente: Requena (2003).

Nota: El estadístico $t$ de Student aparece entre paréntesis debajo del parámetro estimado respectivo.

Nivel de significación:

${ }^{* * *} p<0,001$

${ }^{* *} p<0,01$

${ }^{*} p<0,1$

ción y los ingresos siempre tienen un efecto positivo independientemente de cuales sean las variables por las que se controle el modelo. Pertenecer al sector privado y realizar turnos, o trabajar en empresas de gran tamaño son factores que generan peores niveles de calidad de vida en el trabajo. Lo mismo ocurre con el mayor número de horas laborales semanales y las condiciones de trabajo peligrosas. En el entorno familiar, las responsabilidades familiares, tales como ser cabeza de familia o tener hijos a cargo, suponen peores niveles de calidad de vida en el trabajo. Respecto a los niveles de capital social, todas sus dimensiones son predictores positivos de la calidad de vida en el trabajo. Aun así, las 
variables relativas al capital social son las que más fuerza explicativa tienen en el modelo de regresión sobre la calidad del vida en el trabajo. Esto confirma el hecho de que los procesos que generan relaciones sociales y confianza en los lugares donde las personas interactúan son determinantes para un mayor nivel de calidad laboral, lo que tiene efectos positivos tanto para el individuo como para la institución en la que trabaja.

\section{Conclusiones}

Como se ha podido comprobar, los elementos que componen el capital social son unos buenos predictores de la calidad de vida en el trabajo. En general, actúan como un buen lubricante en el funcionamiento de las instituciones sociales y, como hemos visto, en las económicas. Aunque la ECVT no es una encuesta expresamente diseñada con el objeto de medir el capital social, sin embargo, dada la importancia que el capital social tiene para la conducta del ser humano, ha introducido un buen número de indicadores de capital social que se pueden relacionar con los niveles de satisfacción y con las características de los trabajadores y de las empresas.

En este artículo se ha visto cómo aunque existen diferencias importantes en relación a una completa definición de capital social, es primordial tomar medidas respecto a la importancia de encontrar indicadores empíricos para su medida. De esta forma, aunque existen numerosas encuestas de ámbito supranacional, en las que participa España, que tienen preguntas relativas al capital social (WVS ${ }^{8}, \mathrm{EVS}^{9}$, ISSP ${ }^{10}, \mathrm{ESS}^{11}$, etc.), todavía existen problemas de comparación internacional, para ello, dada la importancia de la cuestión, sería una buena propuesta que, auspiciado por organizaciones supranacionales, tales como la OCDE, Naciones Unidas, Eurostat, etcétera, se normalice la medida de las dimensiones del capital social y se diseñe un cuestionario normalizado entre los diversos países, de forma que se puedan realizar comparaciones entre naciones. Estas comparaciones permitirán aumentar la dimensión correcta de la relación entre capital social, desarrollo e inclusión social, según los diferentes países.

Finalmente, se ha demostrado que el capital social es un asunto socialmente muy importante para el bienestar y la calidad de vida de las personas, en el caso anterior se ha demostrado para la situación laboral, pero esta situación se puede extrapolar para la mayoría de los entornos en los que interactúan los individuos. Asimismo, se ha demostrado que el capital social puede verse como un indicador tanto de bienestar personal como de bienestar social.

8. World Values Surveys.

9. European Values Surveys.

10. International Social Survey Program.

11. European Social Surveys. Su primera edición se centra fundamentalmente en el capital social. 


\section{Bibliografía}

BeugelskijK, S.; Groot, H.L.F.; SCHAiK, A.B.T.M.V. (2002). Trust and economic Growth. Tinbergen University: Tinbergen Institute Discussion Paper, TI 2002-049/3.

BREHN, J.; RAHN, W. (1997). "Individual-Level evidence for the causes and consequences of social capital». American Journal of Political Science, 41, p. 999-1023.

BURT, R. (2000). "The network structure of social capital». En SUTTON, R.I.; STAW, B.M. (eds.) Research in organizational behavior. Greenwich, CT.: JAY Press, vol. 22.

- (2001). «Structural holes versus network closure as social capital». En LIN, N.; COOK, K.; BurT, R. (eds.) Social Capital, Theory and Research. Nueva York: Aldine de Gruyter, p. 31-56.

Coleman, J.S. (1988). «Social capital in the creation of human capital». American Journal of Sociology, 94, p. s95-s12.

De GraAf, N.D.; Flap, H.D. (1988). "With a little help from my friends”: Social resources as an explanation of occupational status and income in West Germany, The Netherlands, and the United States». Social Forces, 67(2), p. 452-472.

Fukuyama, F. (1995). Trust: The Social Virtues And The Creation of Prosperity. Nueva York: Free Press.

- (2000). La gran ruptura. Barcelona: Ediciones B.

GRANOVETTER, M.S. (1985). «Economic action and social structure: The problem of embeddedness». American Journal of Sociology, 91, p. 481-510.

Herreros VÁzQUEZ, F. (2002). "¿Son las relaciones sociales una fuente de recursos? Una definición de capital social». Papers. Revista de Sociología, 67, p. 129-148.

KNACK, S.; KEEFER, P. (1997). «Does social capital have an economic pay-off? A cross country investigation». Quarterly Journal of Economics, 112-4, p. 1251-1288.

Lin, N.; Cook, K.; Burt, R. (eds.) (2001). "Preface». Social Capital, Theory and Research. Nueva York: Aldine de Gruyter.

Ministerio de Trabajo y Asuntos Sociales (2001). Encuesta de Calidad de Vida en el Trabajo 2001. Madrid: MTAS.

OECD (2001). The Well-Being of Nations. The role of human and social capital. OECD: Center for Educational Research and Innovation.

PuTnam, R. (1993). "The prosperous community: Social capital and public life». The American Prospect, 4 (13), p. 35-42.

- (2000). Bowling Alone. The collapse and revival of American Community. Nueva York: Simon \& Schuster.

Putnam, R.; Leonardi, R.; Nanetti, R.Y. (1993). Making Democracy work. Princenton, Nueva Jersey: Princenton University Press.

Pyke, F.; BeCATtini, G.; SENGENBERGER, W. (1990). Industrial districts and inter-firm co-operation in Italy. Ginebra: ILO.

REQUENA, F. (1991). «Social resources and occupational status attainment in Spain: A cross-national comparison with the United States and the Netherlands». International Journal of Comparative Sociology, 23, p. 233-242.

- (2000). «Encuesta de Calidad de Vida en el Trabajo: Posibilidades para el seguimiento de la situación laboral». Revista del Ministerio de Trabajo, Serie Economía y Sociología, 21, p. 235-245.

- (2003). "Social capital, satisfaction and quality of life in the workplace». Social Indicators Research, 61, 3, p. 331-360.

SAXENIAN, A.L. (1994). Regional Advantage: culture and competition in Silicon Valley and Route 128, Cambridge, MA: Harvard University Press. 